\title{
Est-ce que je sais ce que je sais?
}

\section{Christoph Bosshard}

Dr med., vice-président de la FMH, responsable du département Données, démographie et qualité / Académie suisse pour la qualité en médecine (ASQM)

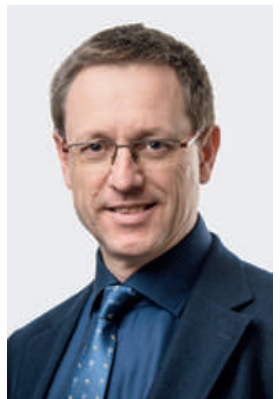

«La seule chose que je sais, c'est que je ne sais rien.» Cicéron attribuait ces paroles au philosophe grec Socrate. Mais en sommes-nous vraiment certains? Loin de nous l'idée de dresser une vue d'ensemble exhaustive de l'éternel débat sur les limites de nos connaissances cela dépasserait clairement le cadre du présent éditorial. En revanche, il est essentiel que nous, médecins, nous interrogions sur nos propres connaissances en portant au quotidien un regard critique d'une part sur la définition de la médecine fondée sur les preuves, et de l'autre, sur les besoins de nos patients, dans un contexte de vieillissement de la population et de polymorbidité accrue - besoins qui vont souvent bien au-delà de la médecine à proprement parler.

Nous ne reviendrons pas ici sur le fait que l'activité médicale doit parfois aussi pouvoir prendre ses distances avec l'évidence scientifique (cette question a déjà fait l'objet de nombreuses discussions), ni sur le fait que $60 \%$ à $70 \%$ des déterminants de santé relèvent de facteurs environnementaux et comportementaux. Mais à l'instar de l'alpiniste qui vérifie son équipement avant de monter au sommet, ou du pilote qui contrôle son

\section{Nous avons nous aussi une part de} responsabilité dans l'application de l'évidence scientifique.

avion avant le décollage, nous devons nous aussi nous interroger sur la fiabilité des fondements scientifiques de notre activité. Nous avons nous aussi une part de responsabilité dans l'application de l'évidence scientifique, que ce soit à notre niveau en tant qu'individu, au niveau des organisations médicales ou au niveau de la société. Et à ce titre, le devoir qui nous incombe nous oblige clairement à faire preuve là aussi d'esprit critique.
La nécessité et la légitimité de cette analyse critique sont au cœur du document de référence et de la prise de position de la FMH présentés dans ce numéro du Bulletin des médecins suisses. Cette année, le congrès annuel de la Société suisse de traumatologie et de médecine des assurances a été l'occasion de prendre connaissance d'exemples éloquents du temps que cela peut durer avant que l'évidence scientifique ne trouve

\section{Nous ne devons pas non plus oublier}

la question de l'applicabilité de la preuve.

sa place dans les guides de pratique médicale (guidelines), ou ne soit mise en application au quotidien. Trop longtemps le syndrome coronarien aigu a été traité à la lidocaïne injectable en faisant confiance au cathétérisme cardiaque droit, alors que l'évidence scientifique indiquait déjà d'autres pistes. Mais il serait réducteur de mettre toute la faute sur la recherche ou sur le retard, inhérent au système, dans la consolidation de la médecine factuelle sous la forme de guides de pratique. Soyons honnêtes, les habitudes et les intérêts ont parfois aussi un rôle à jouer.

Dans notre analyse, nous ne devons pas non plus oublier la question de l'applicabilité de la preuve. Si les essais cliniques randomisés ont une validité intrinsèque élevée, nous avons cependant aussi besoin de données longitudinales et donc de registres. Dans ce domaine, beaucoup a déjà été fait, à l'initiative de différents acteurs et principalement du corps médical. Mais beaucoup reste à faire avant de pouvoir répondre à la question de l'efficacité, de l'adéquation et de l'économicité de l'évidence des guides de pratique.

Je vous remercie pour le soutien et l'esprit critique dont vous faites preuve également sur cet aspect de notre activité! 